\title{
La aposición como estrategia parafrástica
}

\author{
Vanina Andrea Barbeito* \\ Universidad de Buenos Aires, Argentina
}

\begin{abstract}
Resumen
Las gramáticas suelen postular una relación de correferencia entre los constituyentes de la construcción apositiva, lo que permitiría la permutación de los constituyentes en los casos de aposición explicativa. Basados en esta relación de correferencia, algunos autores suelen asociar la aposición con la paráfrasis, operación usualmente definida como el procedimiento de (re)organización del discurso que permite volver sobre un segmento anterior y presentarlo de manera distinta. Desde el Enfoque CognitivoPrototípico, en estudios previos (Barbeito 1999 y 2007a) mostramos la falta de sustento discursivo de la noción de correferencia y de las pruebas de permutación y omisibilidad. Sostuvimos que la relación apositiva resulta de un muy alto grado de continuidad/coherencia entre los conectados y que el hablante realiza en la aposición una operación de (re)conceptualización del designado en la que aparece activamente. En el presente trabajo, nos proponemos establecer las características que asemejan la construcción apositiva a la paráfrasis a partir del análisis cualitativo de un corpus de habla oral espontánea. Consideramos que la relación apositiva emerge del discurso y responde a distintos grados de coherencia entre sus constituyentes. Asimismo, que la aposición resulta ser una gramaticalización de estrategias discursivas parafrásticas prototípicas.
\end{abstract}

* Para correspondencia, dirigirse a: Vanina Andrea Barbeito (vaninabarbeito@filo.uba. ar), Universidad de Buenos Aires, 25 de Mayo 221, Buenos Aires, Argentina. 
Palabras clave: aposición, reformulación, paráfrasis, coherencia, contínuum.

APPOSITION AS PARAPHRASED STRATEGY

\begin{abstract}
Traditional grammarians have pointed out a co reference relation between the constituents in apposition. They argue that this semantic equivalence allows the two component structures to be easily reversible. Some authors tend to associate apposition with paraphrase, which is usually defined as a restatement of the meaning of a text or passage using other words. Within the framework of the CognitivePrototype Theory, we have stated in previous studies (Barbeito, 1999 and 2007a) that speakers use apposition to (re)conceptualize in a different way an entity already mentioned. Also that apposition shows a great degree of coherence between its component structures. This paper deals with a qualitative description of semantic, pragmatic and syntactic features of apposition in order to establish the relation between apposition and paraphrase. We argue that apposition is a grammaticalization of prototypical paraphrasing strategies.
\end{abstract}

Key words: apposition, reformulation, paraphrase, coherence, contínuum.

Recibido: 22/04/13

Aceptado: 03/05/13

\title{
INTRODUCCIÓN
}

Tradicionalmente, las gramáticas han sostenido que entre los constituyentes de la construcción apositiva se establece una relación de correferencia, puesto que ambos constituyentes designan el mismo objeto. Basados en esta relación de equivalencia semántica entre los dos (o más) miembros de la construcción, algunos autores suelen asociar, explícita o implícitamente, la aposición con la paráfrasis.

Desde el Enfoque Cognitivo- Prototípico, marco teórico en el que se encuadra nuestro trabajo, consideramos que la sintaxis está motivada por los objetivos comunicativos y que las relaciones sintácticas reflejan el grado de 
continuidad/coherencia entre los conectados. En este sentido, consideramos que la noción de idéntico referente propuesta por las gramáticas resulta insuficiente para aludir a la influencia de factores de orden semánticopragmático, no solo porque los términos no muestran identidad referencial en todos los casos, sino porque ya no podemos hablar de objeto referido, sino de una conceptualización y (re)conceptualización del designado en ambos miembros de la construcción apositiva.

Dados estos presupuestos, en trabajos anteriores (Barbeito 2007b y 2012) postulamos que el objetivo comunicativo perseguido por el hablante con la aposición es aparecer activamente en la (re)construcción del designado. Teniendo en cuenta esta afirmación y ante la importancia de determinar la motivación semántica, discursiva y pragmática de la aposición, en el presente trabajo nos proponemos estudiar las relaciones entre la función apositiva y la paráfrasis.

Luego de una breve presentación del marco teórico, desarrollaremos seguidamente el estado de la cuestión y resumiremos los resultados de los estudios previos realizados sobre el tema. A continuación, describiremos el corpus y la metodología empírica que se deriva de los principios del enfoque teórico aquí adoptado. Luego, expondremos los resultados y el análisis del corpus. Finalmente, presentaremos las conclusiones.

\section{BREVE PRESENTACIÓN DEL MARCO TEÓRICO}

La revisión crítica de diversas descripciones propuestas para la construcción apositiva en la tradición gramatical española, en estudios funcionalistas y en perspectivas que se reconocen como cognitivo-discursivas, nos permite establecer un conjunto de problemas en el alcance de las descripciones, así como una falta de consistencia entre las definiciones y los ejemplos que las acompañan o que quedan fuera de la descripción.

Desde el Enfoque Cognitivo Prototípico en el que se inscribe este trabajo, sostenemos que los límites de las descripciones propuestas son dependientes, por un lado, de la concepción del signo lingüístico (arbitrario y autónomo) sobre la cual las están construyendo, y, por otro, de la concepción de la gramática como componente autónomo del sistema de la lengua separado del discurso y de otras capacidades cognitivas. Asimismo, dichas descripciones están basadas en un modelo aristotélico clásico de categorización en términos de condiciones necesarias y suficientes que supone la equivalencia lógica de 
los elementos de una categoría. Estas gramáticas descansan además sobre una concepción referencial del significado.

El Enfoque Cognitivo-Prototípico (cfr. Lakoff 1987; Langacker 1987 y 1991, entre otros) no concibe la categorización como un producto arbitrario o un accidente histórico, sino como el resultado de principios psicológicos de categorización (Rosch 1978). En este sentido, el proceso de categorización solo se conoce desde el uso, todo concepto es contextualmente dependiente, los criterios clasificatorios no son seleccionados arbitrariamente en beneficio de un sistema casi preestablecido, sino que se trata de develar los atributos más y menos salientes del elemento en cuestión a partir del presupuesto de que los atributos no deben estar todos presentes en todos los miembros de la categoría en la misma medida. Si las categorías representan mentalmente el conocimiento de la forma de uso de un objeto, el conocimiento tiene una estructura no arbitraria sino motivada por el uso del objeto en situaciones reales, y se sostiene así que los procesos lingüísticos responden a estrategias semejantes a las que rigen los otros procesos mentales, es decir, que no tienen naturaleza modular.

A partir de esta concepción de la categorización surge una concepción de la gramática como producto de la comunicación y de la comprensión y no como fuente o condición previa. Tal como propone Hopper (1998), se plantea un desplazamiento de la gramática del centro a la periferia de la comunicación lingüística y se la considera emergente. La tarea del lingüista para establecer la Gramática Emergente será estudiar las recurrencias de una forma o construcción en el discurso y buscar regularidades que permitan constituir subsistemas.

El trabajo que aquí se propone se enmarca en la visión de la Gramática Emergente del Discurso y recupera, como base teórica, los principios de categorización no discreta ya mencionados. Desde la perspectiva de una Gramática Cognitiva Prototípica -que toma como postulado la motivación semántico-pragmática de los ordenamientos sintácticos- se sostiene que las unidades lingüísticas están agrupadas en conjuntos o categorías que tienden a mostrar un ordenamiento no discreto. La Sintaxis es síntoma del objetivo comunicativo, de aquello que el hablante quiere lograr/ decir, y las construcciones sintácticas deben ser consideradas necesariamente a la luz de los objetivos que se quieren lograr al usarlas (Winters 1990) y son más o menos prototípicas. Se sostienen, entonces, como supuestos teóricos del presente trabajo que las relaciones entre cláusulas son reflejo de la coherencia (continuidad) del discurso, que las categorías tienden a ser no-discretas y que la descripción de las formas y construcciones debe hacerse en función de los contextos reales de uso real. 


\section{ESTADO DE LA CUESTIÓN}

\subsection{APOSICIÓN Y CORREFERENCIA}

Como mencionamos previamente, la aposición ha sido tradicionalmente considerada una relación que consiste en dos unidades correferenciales, es decir que, aunque lingüísticamente diferentes, confluyen en la designación del mismo objeto (Hockett 1955, RAE 1973; de Paula Pombar 1983; Quirk et al. 1985; Kovacci, 1991; A. Di Tullio, 1997; Acuña-Fariña, 2006a y b, entre otros). En este sentido, Martínez sostiene que es la construcción apositiva la que permite que ambos componentes sean considerados correferenciales:

La correferencia característica de la aposición es un contenido expresado en una construcción peculiar, y no algo derivado de la simple reunión de unos determinados lexemas [...] es un valor de contenido realizable, al margen de que le corresponda o no una realidad constatada (Martínez 1985: 455).

Desde un enfoque de corte discursivo, López García (1998) sustenta esta correferencialidad en el hecho de que el puesto y el apuesto conforman una misma escena perceptiva. Dicha percepción, asegura, se origina en la medida en que el apuesto reitera el puesto, con el cual comparte la referencia y la distribución sin más que añadirle algún aspecto diferencial.

A partir del trabajo con un corpus de lengua oral en inglés, Meyer sostiene que la correferencia no siempre se da de manera estricta en la aposición, aunque señala que lo más frecuente es que haya correferencialidad entre los constituyentes, es decir, que ambos refieran a "la misma porción de realidad" (1992: 79).

Desde el enfoque cognitivo, Taylor acuerda con la idea de que en una relación apositiva cada componente designa la misma entidad, pero de diferente manera o con diferentes grados de especificidad, aunque toma cierta distancia de las gramáticas al afirmar que ambos términos se combinan para formar "una concepción más elaborada de la entidad" (2003:235).

La noción de correferencialidad subyace a la distinción que los autores realizan entre la aposición especificativa o restrictiva y la aposición explicativa o no restrictiva, teniendo en cuenta si la segunda unidad de la construcción restringe o explica la referencia de la primera unidad. Gili Gaya indica que en la aposición explicativa el sustantivo complementario "nada añade a la idea que tenemos formada del nombre a que se refiere", sino que "se limita a hacer resaltar una nota o aspecto que nos parece característico 
o particularmente interesante" (1955: 189). Del mismo modo, el Esbozo de la RAE señala:

Cuando decimos Madrid, capital de España no expresamos dos objetos distintos sino uno solo, que viene ya indicado por el primer nombre, Madrid, al cual añade el segundo, capital, otra denominación que explica más el concepto del primero, pero sin precisarlo ni determinarlo distinguiéndolo de otros (1973: 401).

Por su parte, la Nueva Gramática de la Lengua Española de la RAE sostiene que las aposiciones explicativas representan un grupo nominal parentético (en el sentido de 'situado en un inciso') que agrega alguna precisión o algún comentario al contenido del primer miembro de la construcción (2009: 231).

Martínez (1985: 456) considera que tanto en las aposiciones especificativas o unimembres como en las explicativas o bimembres hay especificación recíproca de los términos, es decir, que ambos colaboran en hacer que la referencia del grupo sea más precisa y restringida que la de cada miembro por separado. Concluye que serían explicativas las aposiciones con términos referencialmente igualados (ambos denotadores), como 'Madrid, la capital de España', mientras que las especificativas contarían con términos referencialmente jerarquizados (uno denotador y otro clasificador), como 'El novelista Delibes'.

A diferencia de los autores mencionados, Burton-Roberts (1975) sostiene que no existe la aposición restrictiva y que la aposición propiamente dicha es la tradicionalmente denominada no restrictiva. El autor señala que en una estructura restrictiva como 'The poet Burns' poet y Burns no son correferenciales, puesto que uno de los dos términos no tiene implicancia en la referencia, sino que tiene una función atributiva, de modo que la relación que se establece entre ellos es la de núcleo y atributo (1975: 395). Es decir, plantea que el supuesto de correferencia entre nominales es incompatible con este tipo de construcciones.

Kovacci tampoco considera que las construcciones restrictivas constituyan ejemplos de aposición y denomina 'apositiva' a la tradicionalmente llamada 'explicativa', para cuyo reconocimiento se basa en la configuración prosódica de la construcción (1991: 54-57). Del mismo modo, López

\footnotetext{
El autor incurre aquí en una contradicción, dado que incluye en este último grupo las construcciones compuestas por nombres propios, del tipo Pedro el Cruel, y dice que en estos casos no habría jerarquía referencial entre los términos, siendo que se advierte que el segundo constituyente está, de alguna manera, clasificando al primero.
} 
García (1998) llama aposiciones propias a las que se dan como resultado de la adjunción de una frase nominal entre pausas, el apuesto, a otra frase nominal correferencial, el puesto, y denomina aposiciones aparentes a las construcciones especificativas del tipo 'el rey Felipe II', a las que considera fórmulas fijadas muy próximas a la lexicalización.

\subsection{El CONCEPTO DE PARÁFRASIS}

La noción de paráfrasis se inscribe dentro de las operaciones de reformulación, procedimiento de organización del discurso que tradicionalmente ha sido considerado aquel que permite al hablante volver sobre un segmento anterior para reinterpretarlo y presentarlo de una manera distinta (Garcés Gómez 2006: 654). Dentro de la propuesta de Gülich y Kotschi (1985 y 1995), la reformulación se caracteriza como un proceso de organización discursiva que tiene un carácter interactivo, dado que su función es la de resolver problemas de intercomprensión entre los interlocutores y la de guiar su relación interpersonal.

Según Gülich y Kotschi, en la reformulación la actividad del hablante consiste en caracterizar retrospectivamente una expresión producida anteriormente como insuficiente e identificar una perturbación. Para resolver los problemas así indicados, el hablante tiene procedimientos (o métodos) a su disposición que le permiten modificar, afirmar de modo más preciso, explicar o corregir el segmento de habla que está especificando como expresión de referencia (1995: 39).

La caracterización de los procesos de reformulación se ha configurado en torno a dos tipos de relaciones fundamentales: parafrástica y no parafrástica. Para Gülich y Kotschi el acto de paráfrasis constituye uno de los prototipos del grupo de actos de composición textual (1985: 30). Sostienen que parafrasear es ante todo producir un enunciado de la forma $x R y$, donde $x \mathrm{e}$ $y$ son dos segmentos de la estructura proposicional del texto, y donde R es una relación semántica. En este acto de paráfrasis se manifiestan entonces dos segmentos que los autores llaman enunciado fuente y enunciado reformulador.

Dentro de la reformulación parafrástica, Gülich y Kotschi distinguen la repetición de la paráfrasis propiamente dicha (1995: 50). Señalan que las repeticiones usualmente ocurren sin ningún marcador segmental, mientras que para las paráfrasis existe un conjunto bien caracterizado de marcadores que no son, sin embargo, indispensables para el señalamiento y la interpretación del procedimiento como paráfrasis. Según los autores, la reformulación parafrástica generalmente está ligada a la existencia de 
una equivalencia semántica entre dos enunciados del discurso (1985: 38). Dado que la naturaleza semántica de la relación entre los constituyentes de la paráfrasis puede variar de una ocurrencia a otra, los autores admiten que existe una relación de equivalencia, no solo entre enunciados que tienen una estructura sémica idéntica, sino también entre enunciados donde la semejanza de semas comunes se encuentra más o menos reducida. Esto quiere decir que en muchos casos la equivalencia se funda en una mezcla de rasgos connotativos y denotativos.

La relación de equivalencia planteada en la paráfrasis puede, entonces, variar considerablemente según la proporción entre esos rasgos y puede presentarse de diferentes modos como expansión, reducción o variación: a) expansión, cuando la expresión reformulada es más amplia semánticamente que la expresión de referencia; b) reducción, cuando los rasgos semánticos de una unidad semántica más compleja son "condensados" en la expresión reformulada; y c) variación, cuando los rasgos semánticos de ambos constituyentes son equiparables. Dentro de la expansión, Gülich y Kotschi (1985: 40) incluyen los procedimientos de expansión definitoria y de ejemplificación, mientras que dentro de los procedimientos de reducción incluyen la denominación y el resumen.

La noción de reformulación no-parafrástica, propuesta por Roulet en el marco del análisis del discurso, se define como un tipo de relación interactiva, no argumentativa en la que el enunciador presenta la intervención principal como una nueva formulación ligada a un cambio de perspectiva enunciativa, indicado por el conector, de un movimiento discursivo o de un implícito (1987:115). El autor considera que se establece una subordinación jerárquica del segmento de referencia respecto del segmento reformulado y, de ese modo, la distingue de la reformulación parafrástica. Siguiendo la línea de Roulet, Gülich y Kotschi (1995: 44) afirman que la reformulación no parafrástica se caracteriza por el cambio de perspectiva enunciativa entre la expresión de referencia y la reformulada y el proceso de distanciamiento que ese cambio conlleva, y sostienen que la presencia de marcadores es obligatoria en este tipo de reformulación, puesto que son los encargados de establecer los diferentes tipos de relaciones entre los miembros. Dentro de la reformulación no parafrástica, postulan la existencia de disociaciones y correcciones.

La secuencia repetición-paráfrasis-disociación-corrección propuesta por Gülich y Kotschi (1995: 50) se caracteriza por un incremento en el grado de distancia entre la expresión de referencia y la reformulada. Mientras las repeticiones no expresan distancia, las paráfrasis generalmente expresan una distancia débil y las disociaciones contienen varios grados de distancia, 'débil', 'media' o 'fuerte', pero siempre por debajo del nivel de la invalidación, que se reserva para las correcciones. 


\subsection{ApOSICIÓN Y PARÁFRASIS}

De acuerdo con la propuesta sobre reformulación de Gülich y Kotschi, que es considerada por los teóricos una de las más completas hasta el momento, pueden establecerse características propias tanto para la reformulación parafrástica como para la no-parafrástica. Vimos en el apartado previo que en el caso de la reformulación parafrástica, se plantea una relación de equivalencia entre los dos segmentos que puede ser más fuerte o más débil dependiendo del grado de compatibilidad semántica entre los constituyentes enlazados.

Observamos que el criterio utilizado para determinar si una expresión puede considerarse paráfrasis de A es semántico. En este sentido, si B es semánticamente equivalente a $\mathrm{A}, \mathrm{B}$ se entiende como un cuasi-sinónimo o una definición de A. Como se predica una equivalencia, y por lo tanto, una posible sustitución, se considera a la paráfrasis un hecho de lengua cercano a la sinonimia.

Esta concepción del fenómeno se sustenta, por un lado, en que el significado y el referente se identifican, puesto que se defiende que los enunciados parafrásticos aportan generalmente la misma información del referente, pero con un valor explicativo (Fuchs 1994). Probablemente esta equiparación entre significado y referente provenga de la paráfrasis explicativa del discurso científico, en la que se suele establecer una sinonimia referencial entre términos científicos y términos comunes. Sin embargo, como señala Galán Rodríguez (1998: 91), aun en estos casos la paráfrasis es frecuentemente una reducción de significado y no una equivalencia, porque los dos términos no pertenecen al mismo paradigma y la paráfrasis se convierte en explicación.

Por otro lado, si la paráfrasis se funda en una equivalencia semántica habría que considerar la posibilidad de considerar a los términos A y B elementos intercambiables. Sin embargo, esta inversión del orden basada en una supuesta equivalencia de la designación y/o del sentido puede acarrear diferencias expresivas (Casado 1991:108) y discursivas.

Esta supuesta equifuncionalidad de los dos miembros y el valor explicativo que se le asigna al segundo enunciado suelen favorecer la equiparación con la función de aposición explicativa. Así, De Paula Pombar (1983: 53) establece un grupo especial en el que las aposiciones pueden ir precedidas por introductores que reflejen la identidad de referente, del tipo $o$ sea, es decir, por ejemplo, etc. Del mismo modo, Hernández (1984: 429) señala que cualquier adyacente equifuncional, incluyendo los introducidos por un conector gramaticalizado como o sea, a saber, es decir, que incida sobre un sintagma nominal como desarrollo o expansión, iría en aposición explicativa. 
Meyer (1992: 79) establece tres clases semánticas de aposición: a) clases semánticas donde el segundo miembro da información más específica sobre el primero, entre las que cuenta la identificación, apelación, particularización y ejemplificación; b) clases semánticas donde el segundo miembro da una información menos específica sobre el primero, como la caracterización; y c) clases semánticas donde el segundo miembro da una información equivalente a la del primero, dentro de las cuales incluye la paráfrasis, la reorientación y la autocorrección.

Del mismo modo, Suñer Grataços considera la paráfrasis como la operación que está en el seno de la aposición explicativa, no restrictiva o descriptiva, a la que define como aquella que explica "mediante una paráfrasis nominal alguna de sus propiedades intrínsecas" (1999: 542).

\subsection{El CONTÍNUUM DE RELACIONES SINTÁCTICAS}

En el marco de una sintaxis motivada, que refleja la continuidad del discurso, las categorías sintácticas y semánticas no son necesariamente discretas. En este sentido, Givón (1984) interpreta el espacio nocional cognitivo como un espacio no discreto, como un contínuum en el que también las categorías forman un contínuum.

Al tratar las relaciones adverbiales, Givón basa su análisis en el siguiente principio de iconicidad: cuanto más integrados semántica/pragmáticamente están dos hechos/estados, más integradas gramaticalmente estarán las cláusulas que los codifican. En este sentido, sostiene que en muchas lenguas los hechos que subyacen al principio de iconicidad no soportan una separación clara entre subordinación y coordinación, y revelan la existencia de una escala graduada de integración clausal. Señala como componentes de integración aquellos que hacen a la continuidad temática: continuidad referencial, temporal, de locación y de acción.

En el marco de una sintaxis motivada, que refleja la continuidad del discurso, lo que implica que la continuidad sea una cuestión de grados, las categorías sintácticas y semánticas no son necesariamente discretas. Reconoce que las categorías naturales cognitivas, así como las lingüísticas, no siempre se definen en términos de un criterio simple o de un conjunto de propiedades identificatorias (suficientes y necesarias) a la manera clásica $^{2}$. Para Givón, las categorías están formadas por intersecciones de un

2 Desde Aristóteles en adelante, prácticamente todas las teorías que se ocupan de la categorización se basan en que el proceso de categorización se lleva a cabo a través de unidades 
número de características o rasgos que tienden a coincidir estadísticamente/ probabilísticamente, pero que no coinciden siempre de manera absoluta. Se identifican así aquellos miembros que gozan de todas las características como miembros prototípicos, como "mejores ejemplos" de la categoría que otros, y se permite hablar de centralidad y gradación ${ }^{3}$.

Dentro de esta línea, Borzi (1995 y 2001) postula un contínuum de relaciones sintácticas entre cláusulas que reflejan los grados de continuidad del discurso y que quedan caracterizadas a partir de una sumatoria de atributos. Considerar las relaciones sintácticas en este tipo de contínuum, que es ordenado y no discreto, permite integrar sistemáticamente todos los ejemplos que se van a organizar a partir del grado de continuidad discursiva del que deriven.

En la zona de la izquierda de este contínuum se encuentra la subordinación de actante, que se corresponde con un alto grado de continuidad: las cláusulas comparten tema y función retórica, el evento de una de las cláusulas es parte del evento de la otra y el orden de las cláusulas refleja relativamente el orden de los eventos. La información vieja está en la cláusula introducida por un encabezador (tematizador) y esta es la cláusula que se describe como subordinada. La información nueva está en la cláusula que se describe como principal. En el otro extremo de este contínuum (ubicado hipotéticamente a la derecha) se presenta la subordinación retórica (o centro-periferia), en la que el orden de las cláusulas no refleja necesariamente el orden de los eventos. El objetivo del hablante es presentar dos cláusulas con distinto objetivo comunicativo o distinta función retórica. La información nueva ocupa la segunda posición y está señalizada por un encabezador (focalizador). En términos de relación sintáctica es una zona que comparte atributos tanto con la bipolaridad como con la concepción figura/fondo.

Ubicadas dentro de una hipotética ojiva, estas dos relaciones sintácticas, extremos del contínuum, confluyen en sus arcos en la relación de coordinación. En esta relación, lo que prevalece es el equilibrio pragmático, semántico y sintáctico con el único desequilibrio del orden de las cláusulas que es icónico respecto del orden de los eventos, se conectan dos eventos

discretas y que estas categorías son divisibles y formalizables a través de un conjunto de rasgos definitorios, en función de los cuales también se determina su pertenencia o no a una categoría. Este enfoque implica que: a) las categorías quedan definidas por un conjunto de propiedades necesarias y suficientes; b) estas propiedades/características son binarias; c) las categorías tienen límites precisos; d) todos los miembros de la categoría tienen igual importancia, o son representantes equivalentes de la categoría en cuestión.

Para un mejor desarrollo de la noción de prototipo, confróntese Rosch (1978). 
de igual importancia, los actantes no pueden coincidir en el designado y coherentemente con esto se espera que en ambas cláusulas se presente información nueva. El conector ocupa posición fija entre los conectados.

A partir del planteo inicial de Givón y de la propuesta de ordenamiento de las relaciones sintácticas de Borzi, consideramos como presupuesto del presente trabajo que la relación apositiva emerge del discurso y responde a distintos grados de coherencia entre sus constituyentes.

\section{RESULTADOS PREVIOS}

Las gramáticas han propuesto la noción de correferencia entre los términos de la construcción apositiva, equivalencia significativa que permitiría la permutación y omisibilidad de los constituyentes. Los autores deducen tales definiciones de correferencialidad a partir de ejemplos como 'Estuvimos con Don Enrique, el tío de Juan', 'la ciudad de Córdoba' o 'el poeta Neruda'. A la puntual crítica de Burton-Roberts (1975) de inexistencia de correferencialidad para los casos restrictivos, en trabajos anteriores (Barbeito 2004 y 2007a) mostramos que en estructuras como 'Se está tratando de productos alimenticios, harinas, aves, conejos, derivados del maíz', los llenados léxicos de ambos constituyentes no denotan la misma realidad extralingüística, ni connotan los mismos significados. Las relaciones de significación entre los términos se muestran en estos casos dependientes del contexto discursivo que las enmarca, de modo tal que la noción de 'idéntico referente' resulta insuficiente para aludir a la influencia de factores de orden semántico-pragmático.

A su vez, en Barbeito (2007b) señalamos que los términos en aposición no muestran identidad referencial en todos los casos, porque desde el enfoque cognitivo ya no podemos hablar de 'objeto referido', sino de una conceptualización del objeto, evento o acción dentro de ciertas circunstancias que incluyen factores de relevancia, relaciones sociales, desarrollo del discurso y habilidades lingüísticas ${ }^{4}$. En este sentido, planteamos que el

$4 \quad$ Langacker (1987) señala que cuando el hablante tiene que encontrar una expresión lingüística para una conceptualización, la situación instaura una variedad de constricciones (constraints) sobre cuál es la solución aceptable. Esas constricciones incluyen factores como: cuántos detalles el hablante considera relevantes, qué aspectos de la conceptualización quiere 
objetivo comunicativo perseguido por el hablante con la aposición no es solo añadir datos informativos sobre la entidad ya designada, sino transmitir la elección de un significado en particular por sobre otros significados en el proceso de (re)construcción del objeto dentro del discurso. En síntesis, cuando el hablante apone un elemento a otro selecciona una parte saliente de una entidad para conceptualizar el participante re-designado. Esos resultados previos nos llevaron, por un lado, a jerarquizar un atributo como fundamental en la construcción apositiva: la coincidencia de designado entre ambos constituyentes. Por otro lado, nos permitieron descartar las pruebas de permutación y de omisibilidad de los constituyentes, ya que, desde un punto de vista discursivo, no tienen ningún sustento, en tanto todo lo que dice el hablante y tal como lo dice es funcional al significado del discurso.

\section{PROBLEMAS E HIPÓTESIS}

Del análisis crítico del estado de la cuestión se concluye que los autores tienen en mente, al clasificar los fenómenos discursivos, las características definitorias de aposición que las gramáticas han sostenido hasta el momento en un movimiento inverso al que sostiene el Enfoque Cognitivo, de proyección de los fenómenos oracionales al discurso. Asimismo, coinciden en la presencia de equivalencia semántica entre los miembros en aposición, relación que, como vimos en $\S 2.2$, también se postula para los miembros de la paráfrasis o de la reformulación no-parafrástica.

Cuando los teóricos hablan de correferencia en la aposición, están pensando en dos segmentos que se refieren a una misma realidad, pero no señalan que ambos deban compartir significado, como sí proponen cuando hablan de paráfrasis. Tampoco distinguen la identidad referencial extralingüística de la identidad de designado creada por el hablante entre ambos segmentos en el discurso.

Por otra parte, la aposición queda definida por los autores como una relación sintáctica del dictum (Fuentes Rodríguez 1989: 231), mientras

enfatizar, su relación social con el oyente, su conocimiento sobre cuánto conoce el oyente del contexto y la noción que va a comunicar, cómo se integra la noción con el discurso previo, el efecto que quiere causar en el oyente, su estimación respecto de la habilidad lingüística del oyente y cuánto desea desviarse de la convención lingüística. 
que la explicación, operación con la que implícita o explícitamente suele asociarse la aposición, suele considerarse una función de nivel enunciativo en la que el hablante se manifiesta como constructor de la comunicación y tiene una posición intradiscursiva.

Los resultados de nuestros trabajos previos permiten concluir que la aposición, entendida como una actividad lingüística de explicación, recorre todos los planos lingüísticos, dado que el hablante se revela como el responsable de la creación de ciertas equivalencias discursivas, al margen de su correferencia o correspondencia con la realidad ${ }^{5}$.

En virtud de los resultados previamente reseñados, consideramos que las atribuciones y asociaciones de significado son arbitrarias (ocasionales, discursivas) e intencionales, ya que no parten de una relación sinonímica sino de un punto de vista sobre las cosas. En este sentido, consideramos que no podemos hablar en términos semánticos de una relación de equivalencia entre los constituyentes, dado que toda reformulación está orientada y establece una equiparación dinámica y extremadamente variable entre los miembros.

Dados estos presupuestos, en el presente trabajo analizamos la construcción apositiva sobre un corpus oral de habla espontánea y postulamos que el grado de continuidad/coherencia entre los conectados motiva distintos grados de integración gramatical y que la relación apositiva resulta ser una gramaticalización de estrategias discursivas parafrásticas prototípicas de altísima coherencia entre los constituyentes.

\section{CORPUS Y METODOLOGÍA}

En esta oportunidad, y coherentemente con los principios del enfoque, se ha partido de un corpus auténtico de habla oral espontánea. El corpus está constituido por:

\footnotetext{
5 Ante las dificultades que derivan del término 'paráfrasis' y/o 'aposición explicativa' con nexo, Galán Rodríguez (1998), en un estudio sobre los conectores es decir y o sea, propone reemplazar la denominación de 'marcador de reformulación parafrástica' por el de 'marcador o acotador de posiciones discursivas', puesto que revela "tanto el control que ejerce el emisor sobre la interpretación como la presencia o grado de compromiso del emisor con su discurso" (1998: 93).
} 
- 35 entrevistas periodísticas efectuadas por una periodista para dos medios gráficos durante el período 1998-2006. Los entrevistados son adultos de ambos sexos, con edades que oscilan entre 20 y 70 años, nivel de escolaridad media y/o universitaria y profesiones varias.

- 11 entrevistas realizadas por un grupo de estudiantes del Instituto de Enseñanza Superior en Lenguas Vivas 'Juan Ramón Fernández’ al plantel terciario de la institución para indagar en las representaciones sobre el objeto de enseñanza-aprendizaje entre los docentes.

Sobre el total del corpus transcripto se registraron 500 ocurrencias de aposiciones que sirvieron de base para la descripción. El análisis cualitativo y cuantitativo del cuerpo de datos nos permitió determinar qué tipo de construcciones entran más o menos frecuentemente en aposición. De acuerdo con la constitución interna de cada uno de los constituyentes, sobre el total de ocurrencias del corpus se registraron 228 casos de aposición entre constituyentes nominales (45,60\%), 170 casos de aposición no nominal (34\%) y 102 casos combinados $(20,40 \%)$.

Incluimos dentro de lo que denominamos nominal no solo al sustantivo, sino también al adjetivo, el infinitivo y las construcciones relativas. En las aposiciones no nominales el llenado de ambos constituyentes es no nominal (construcciones prepositivas, adverbios, verbos, verboides, cláusulas), mientras que en los casos combinados uno de los miembros es de naturaleza nominal y el otro, de naturaleza no nominal. Según los datos obtenidos, la aparición de aposiciones nominales es frecuente $(45,60 \%)$ pero no es el tipo de aposición excluyente en el corpus ${ }^{6}$.

Partimos del presupuesto de que la problemática de una relación cercana a la paráfrasis entre los constituyentes apuestos puede darse dentro del continuo en los casos donde encontramos lo que podríamos llamar 'aposiciones entre cláusulas', dado que se trata de casos altamente discursivos en los que se comparan eventos con eventos. En esta oportunidad, analizamos cualitativamente los 61 casos de aposición entre cláusulas registrados, que constituyen el 36\% de los casos de aposición no nominal e incluyen ejemplos como (i):

6 El problema de la aposición no nominal fue analizado en Autor (2001). 
(i) "Teníamos mucha dificultad en llegar al relleno sanitario, nos encajábamos con los camiones ${ }^{7}$. Empezó a haber una alta preocupación en lo que es la contaminación de napas. [...]" $(\mathrm{CD}, 12)$.

\section{ANÁLISIS}

A partir del análisis del corpus hemos podido observar que, si bien la aposición manifiesta un alto grado de coherencia/continuidad entre los miembros, el grado de integración de los constituyentes no es el mismo en todos los casos y que además, no se da equivalencia (como quieren los autores) porque justamente la ausencia de equivalencia es lo que permite establecer que hay un núcleo ${ }^{8}$.

Congruentemente con el modelo teórico adoptado, sostenemos que no todos los atributos deben necesariamente manifestarse en cada una de las instancias de la categoría ni en la misma medida. La zona apositiva presenta límites imprecisos y puede presentar distintos grados de continuidad. En este sentido, el análisis de las relaciones semántico-pragmáticas y sintácticas que se establecen entre las cláusulas en relación apositiva nos permite establecer dos polos en el contínuum de la zona apositiva de acuerdo con el grado de continuidad de los conectados. Uno de estos extremos se corresponde con alta continuidad y el otro con baja continuidad y, a su vez, cada extremo del contínuum aparece como el resultado de la acumulación o la pérdida de distintos rasgos.

Los rasgos a considerar serán los siguientes:

1. Identidad en la modalidad entre ambos miembros de la construcción apositiva.

2. Continuidad tópica: entendida como identidad de sujeto entre las cláusulas con el recurso de la desinencia verbal para recuperarlo en la segunda cláusula

3. Identidad de eventos: entendida como igual lugar, tiempo, acciones que tienden a pertenecer a un mismo script o marco

Señalamos la construcción apositiva en cursiva.

8 Cf. Autor (2006) y Autor (2008) para el análisis del problema del núcleo en la construcción apositiva. 
4. Coherencia temática

5. Identidad de tiempo y aspecto entre los verbos de ambas cláusulas

En un extremo del contínuum mencionado, se ubicarán aquellos casos que presentan:

1. Identidad en la modalidad

2. Alta continuidad tópica

3. Identidad de eventos

4. Mayor coherencia temática

5. Identidad de tiempo y aspecto verbal.

Los ejemplos (ii), (iii) y (iv) ilustran este extremo del contínuum, que representa una relación a la que denominaremos aposición parafrástica:

(ii) Se encaró un proceso muy importante, se construyó el Canal de la Esperanza, se construyó el Canal de la Sarita, se construyó el canal en Moctezuma y se realizaron obras de canalización en la zona de El Villar (CD, 356).

(iii) Lo que pasa es que en el cine los excitan tanto, los vuelven tan locos, que reaccionan $[\ldots](\mathrm{CD}, 437)$.

(iv) Soy licenciado y profesor en ciencias de la educación, tengo los dos títulos (CD, 155).

En el otro extremo del contínuum, se ubicarán aquellos casos que manifiestan:

1. Igual o distinta modalidad

2. Continuidad tópica relativa (con o sin identidad de sujeto)

3. Miembros referidos a igual o distinto evento

4. Menor coherencia temática

5. Igual o distinto tiempo y aspecto verbal:

(v) En Europa estaba todo a disposición mía, era canilla libre [...] (CD, 440).

(vi) Desde que empecé no han cambiado mucho las características: las gomas son las mismas $[\ldots](\mathrm{CD}, 413)$.

(vii) $[\ldots]$ como que agregarle todavía una complicación más en cuanto a teorías del aprendizaje a mí me parecía que desbordaba los objetivos que planteaba para Psico I, quedaban como más confundidos [... $(\mathrm{CD}, 122)$. 
En aquellos casos en los que el grado de continuidad/coherencia entre los miembros es alto nos encontramos frente a casos de aposición parafrástica prototípica, mientras que los casos de baja continuidad/coherencia representan una relación de aposición parafrástica marginal o, en otras palabras, de aposición no parafrástica. En estos últimos casos, el enunciador presenta la aposición como una nueva formulación ligada a un cambio de perspectiva y muestra un movimiento discursivo que se evidencia aún más en la intercalación de conectores de reformulación:

(viii) [...] siempre, en todos los casos, sin excepción las visitas a las provincias las hacemos con nuestro Jefe de Distrito de Vialidad Nacional, es decir, se trabaja en forma conjunta en todos los aspectos (322)

(ix) [...] que sea una empresa que tenga muchas participaciones en el debate público, o sea, que defienda los valores que considera que son buenos para la patria, el esfuerzo, el trabajo, la honestidad, la creatividad, la generosidad, la pasión, el entusiasmo $(\mathrm{CD}, 448)$.

Los casos de baja continuidad entre los conectados responden a cláusulas en las que lo que importa no son los hechos sino para qué los usa el hablante en el discurso y en la situación. El proceso de distanciamiento que se genera entre los constituyentes conlleva, aunque no de forma obligatoria, la presencia de marcadores, puesto que refuerzan los diferentes tipos de relaciones entre los miembros.

En la Figura 1, se presentan las zonas, los rasgos y los ejemplos:

Figura 1: La zona apositiva

+ Continuidad/

-Continuidad/

Coherencia
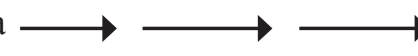

Coherencia

Aposición parafrástica

+Identidad en la modalidad

+ Continuidad tópica

+Identidad de eventos

+Coherencia temática

+Identidad tiempo y aspecto verbal

- Presencia de conector
Aposición no parafrástica

+/- Identidad en la modalidad

+/-Continuidad tópica

+/-Identidad de eventos

+/-Coherencia temática

+/-Identidad tiempo y aspecto verbal

+/-presencia de conector 
[...] estamos poniendo muchos jacarandás, [...] Tenemos una oferta educativa estamos poniendo por todos lados jacarandás $(\mathrm{CD}, 51)$

$[\ldots](\mathrm{CD}, 34)$ muy importante, Pehuajó tiene más de 110 servicios educativos en el distrito

[...] sé que soy una profesora rioplatense, [...] allá tenés todo a dos cuadras, me considero como Natalia Oreiro, soy del Río de la Plata [...] (CD, 97)

Hemos postulado un contínuum de relaciones sintácticas entre cláusulas que reflejan los grados de continuidad del discurso y que quedan caracterizadas a partir de una sumatoria de atributos. Considerar las relaciones sintácticas en este tipo de contínuum, que es ordenado y no discreto, permite integrar sistemáticamente todos los ejemplos que se van a organizar a partir del grado de continuidad discursiva del que deriven.

Tal como se muestra en el contínuum propuesto, en la aposición el productor del discurso ejerce la facultad de hacer como si ambos constituyentes se identificasen semánticamente ("Tenemos una oferta educativa importante, Pehuajó tiene más de 110 servicios educativos.."), aun cuando la continuidad tópica entre ambos constituyentes sea baja. De ese modo, manifiesta su posición discursiva dominante sobre el receptor, quien ha de aceptar o no las equivalencias propuestas.

A partir del análisis del corpus hemos podido observar que el grado de integración gramatical de los constituyentes no es el mismo en todos los casos y que además, no se da equivalencia (como quieren los autores) porque justamente la ausencia de equivalencia es lo que permite establecer que hay un núcleo en la construcción. La idea de equivalencia de los autores está fundada en una supuesta identidad de referente, algo que no se sostiene, tal como afirmamos anteriormente. La relación apositiva emerge del discurso y responde a distintos grados de coherencia entre sus constituyentes, que tienen su correlato en la linealidad del discurso.

El análisis del corpus muestra que las atribuciones y asociaciones de significado entre ambos constituyentes no parten de una relación sinonímica sino de un punto de vista sobre las cosas. En este sentido, consideramos que no podemos hablar en términos semánticos de una relación de equivalencia entre los constituyentes, dado que toda reformulación está orientada y establece una equiparación dinámica y extremadamente variable entre los miembros.

El análisis de las relaciones pragmático-semánticas y sintácticas que se establecen entre los constituyentes de la construcción apositiva nos permite 
mostrar, entonces, que la aposición como la entienden los gramáticos (Cfr. por ej. El Ministro de Economía, Juan Fernández) resulta ser, en todo caso, una gramaticalización de estrategias discursivas parafrásticas prototípicas de altísima coherencia entre los constituyentes.

\section{CONCLUSIONES}

El análisis de las construcciones en un contínuum de mayor a menor coherencia muestra que la reformulación no es simplemente una operación lingüística que se produce para marcar el carácter provisional o inadecuado de un enunciado o de una expresión. Tampoco es solo un índice de los obstáculos que debe superar el emisor en la producción de su discurso para garantizar la comprensión. En el papel metalingüístico de reformulación, presente en la aposición, se evidencia la intervención del hablante, la misma que, para organizar la información, coloca los conectores de reformulación cuando lo cree necesario.

La aposición muestra un alto grado de integración entre los constituyentes, que deriva de la coincidencia en el designado, en el hecho de que uno de los miembros de la construcción suele tener preeminencia sobre el otro en el hilo del discurso. Si bien, tal como señalamos antes, la construcción apositiva muestra un equilibrio entre los constituyentes, y esto se verifica en el hecho de que hay coincidencia de designado, también muestra desequilibrio en el orden de los constituyentes en la linealidad del discurso. Además, constituye un mecanismo de conexión que tiene la posibilidad de intercalar entre los miembros de la construcción, si bien no coordinantes auténticos, marcadores explícitos diversos, entre los cuales se cuentan las formas que quedan clasificadas para las gramáticas entre los conectores de reformulación como 'es decir' y 'o sea'. En consecuencia, se advierten distintos grados de continuidad de los conectados que reciben distintos tipos de marcadores.

Teniendo en cuenta que el hablante usa la aposición para aparecer activamente en la (re)conceptualización de entidades mencionadas en el discurso y de ese modo construir, organizar y (re)orientar la continuidad discursiva, postulamos que la aposición responde a cláusulas en las que lo que importa no son los hechos sino para qué los usa el hablante en el discurso y en la situación. Este atributo se evidencia en nuestra concepción de la aposición como recurso utilizado por el hablante para redefinir el designado, construir y/o (re)orientar la argumentación en el discurso. Creemos que en el papel metalingüístico de reformulación, presente en la aposición, hay un 
movimiento hacia atrás y un retraso en el avance de la información textual, en el que está presente la intervención del hablante, la misma que para organizar la información puede colocar conectores.

\section{REFERENCIAS BIBLIOGRÁFICAS}

AcuÑa-Fariña, Juan Carlos. 2006a. A constructional network in appositive space. Cognitive Linguistics 17-1: 1-37. 33: $29-52$.

2006b. Polycentric apposition in English. Copenhagen Studies In Language

Barbeito, Vanina. 2001. Manifestación de la aposición no nominal. En Eduardo Quiroga Salcedo (coord.). Hispanismo en la Argentina. En los portales del siglo XXI, pp. 107-114. San Juan: Editorial Universidad Nacional de San Juan.

1999. Estudio sobre la aposición. Ponencia presentada en el Congreso Internacional "La Gramática: Modelos, Enseñanza, Historia", Buenos Aires, Argentina. 2004. Manifestación de la aposición no nominal, un enfoque discursivo. Ponencia presentada en el Segundo Coloquio Nacional de Investigadores en Estudios del Discurso, La Plata, Argentina.

2006. El problema del establecimiento del núcleo en la construcción apositiva. Ponencia presentada en el X Congreso Nacional de Lingüística, Salta, Argentina.

2007a. La construcción apositiva: ¿correferencia o (re)conceptualización? En Vanina Barbeito y Laura Miñones (coords.). Problemas de Sintaxis del español. Un enfoque Cognitivo-Prototípico, pp. 29-38. Buenos Aires: Facultad de Filosofia y Letras, Universidad de Buenos Aires.

2007b. Encadenamientos argumentativos y semántica de la construcción apositiva. Ponencia presentada en el III Coloquio Argentino de la IADA: Diálogo y contexto, La Plata, Argentina.

2008. Hablemos de eso, la aposición. En Cristina Messineo, Marisa Malvestitti y Roberto Bein (eds.). Estudios en lingüística y antropología. Homenaje a Ana Gerzsenstein, pp. 341-353. Buenos Aires: Facultad de Filosofía y Letras, Universidad de Buenos Aires. 2012. Para qué usamos la aposición. En Adolfo García (edit.). Aproximaciones teóricas y empíricas a la Lingüistica Cognitiva. Mar del Plata: Editorial Martín.

Borzi, Claudia. 1995. El contínuum de las relaciones sintácticas. Estudios filológicos 30: 29-42. 2001. Coordinación y subordinación: zonas de una ojiva. En Elvira Arnoux y Ángela Di Tullio (eds.). Homenaje a Ofelia Kovacci, pp. 91-111. Buenos Aires: Eudeba.

Burton-Roberts, Noel. 1975. Nominal Apposition. Foundations of Language 123: 391-419.

Casado Velarde, Manuel. 1991. Los operadores discursivos es decir, esto es, o sea y a saber en el español actual: valores de lengua y funciones textuales. LEA XIII: 87-116.

De Paula Pombar, María de las Nieves. 1983. Contribución al estudio de la aposición en el español actual. Verba: Anejo 20.

Di Tullio, Ángela. 1997. Manual de gramática del español. Buenos Aires: Edicial.

Fuchs, CATHERINE. 1994. Paraphrase et énonciation. Paris: OPHRYS.

Fuentes Rodríguez, Catalina.1989. De nuevo sobre la aposición. Verba 16: 215:1-236.

Galán Rodríguez, Carmen. 1998. La dimensión explicativa y deóntica de los conectores $o$ sea y es decir. Anuario de Estudios Filológicos XXI: 85-104. 
Garcés Gómez, María Pilar. 2006. Las operaciones de reformulación. Ponencia presentada en el XXXV Simposio Internacional de la Sociedad Española de Lingüística, León, España.

Gili Gaya, Samuel. 1955. Curso de Sintaxis Española. Barcelona: Spes.

Givón, Talmy. 1984. Syntax. Amsterdam/Philadelphia: J. Benjamins.

Gülich, Elizabeth y Thomas Kotschi. 1985. Les actes de reformulation dans la consultation La dame de Caluire. En Pierre Bange (ed.). L'analyse des interactions verbales. La dame de Caluire: une consultation. Berne : Peter Lang.

1995. Discourse production in oral communication. En Uta Quasthoff (ed.). Aspects of oral communication. Berlin: Mouton de Gruyter.

Hernández, CÉSAR. 1984. Gramática funcional del español. Madrid: Gredos.

Hockett, Charles. 1958. A course in Modern Linguistics. New York: The Macmillan Company.

Hopper, Paul. 1998. Emergent Grammar. En Michael Tomasello (ed.). The new psychology of language. Cognitive and functional approaches to language structure, pp. 155- 175. New Jersey: LEA.

Kovacci, Ofelia. 1991. El comentario gramatical I y II. Madrid: Arco Libros.

Lakoff, George. 1987. Women, fire and dangerous things. Chicago: University Press.

Langacker, Ronald. 1991. Foundations of Cognitive Grammar. Descriptive Applications. Stanford: Stanford University Press, Vol.2.

-1987. Foundations of Cognitive Grammar. Stanford: Stanford University Press, Vol.1

López García, Ángel. 1998. Gramática del español III. Las partes de la oración. Madrid: Arco Libros.

Martínez, José. 1985. Las construcciones apositivas en español. Philologica Hispaniensia II Lingüistica. In honorem Manuel Alvar. Madrid: Gredos.

Meyer, Charles. 1992. Apposition in Contemporary English. Cambridge: Cambridge University Press.

Quirk, Randolph, Sidney Greenbaum, Geoffrey Leech y Jan Svartvik. 1985. A comprehensive grammar of the English Language. London: Longman.

Real Academia Española. 1973. Esbozo de una Nueva Gramática de la Lengua Española. Madrid: Espasa-Calpe.

Real Academia Española y Asociación de Academias de la Lengua Española. 2009. Nueva Gramática de la Lengua Española. Madrid: Espasa-Libros.

Rosch, Eleanor. 1978. Principles of categorization. En Eleanor Rosch y Barbara Lloyd (ed.). Cognition and categorization, pp. 27-47. Hillsdale, NJ: Erlbaum.

Roulet, Eddy. 1987. Complétude interactive et connecteurs reformulatifs. Cahiers de Linguistique Française 8: 111-140.

SuÑer Grataçós, Avellina. 1999. La aposición y otras relaciones de predicación en el sintagma nominal. En Ignacio Bosque y Violeta Demonte (coord.). Gramática Descriptiva de la Lengua Española, pp. 523-564. Madrid: Espasa.

TAYLOR, John. 2002. Cognitive Grammar. Oxford: Oxford University Press.

Winters, Margaret. 1990. Toward a theory of syntactic prototypes. En Savas Tsohatzidis (ed.). Meanings and Prototypes. Studies in linguistic Categorization, pp. 285-306. London: Routledge. 